Marquette University

e-Publications@Marquette

College of Nursing Faculty Research and

Publications

Nursing, College of

$12-1-2005$

Psychometric Evaluation of the Shared Care Instrument in a Sample of Home Health Care Family Dyads

Margaret Sebern

Marquette University, margaret.sebern@marquette.edu

Accepted version. Journal of Nursing Measurement, Vol. 13, No. 3 (Winter 2005): 175-191. DOI. (C) 2005 Springer Publishing Company. Used with permission.

Margaret Sebern was affiliated with the University of Wisconsin-Milwaukee at the time of publication. 


\section{Psychometric Evaluation of the Shared Care Instrument in a Sample of Home Health Care Family Dyads}

By Margaret D. Sebern

Researchers have studied negative effects of caregiving on a family caregiver; however, less is known about positive aspects of exchanging assistance for both members of a family caregiving dyad. In a previous naturalistic inquiry the author indentified a basis for studying caregiving interactions was a construct called shared care. The three components of shared care identified in the naturalistic inquiry were communication, decision making, and reciprocity. The Shared Care Instrument (SCl) was developed to measure the construct. The purpose of this study was to assess the psychometric properties of the $\mathrm{SCl}$, and to assess its construct and criterion-related validity. A sample of home care family dyads (110 patients and 109 family members) returned usable survey questionnaires. Results indicated the Cronbach's alphas for the patient group for the $\mathrm{SCl}$ subscales ranged from .78 to .84 , and .77 to .79 for family members. Factor analysis supported the underlying theoretical basis and factor structure of the SCI. Criterion-related validity was also supported. Therefore, the results of this study provide initial evidence for the reliability and validity of the $\mathrm{SCl}$ for use with family caregiving dyads. The findings support the need for additional testing of the SCI.

A major focus in the elder family care literature has been on caregivers and negative aspects of providing care, with the exclusion of care recipients. Yet by its nature, caregiving is a dyadic process. Several researchers suggested that care recipients not only receive support from, but also provide substantial support to their family, and these acts of reciprocity are important to well-being (Beach, Schulz,Yee, \& Jackson, 2000; Liang, Krause, \& Bennett, 2001). Reinardy and colleagues (Reinardy, Kane, Huck, Call, \& Shen, 1999) pointed out that positive consequences of informal caregiving are under-reported because many instruments measure negative aspects of caregiving (e.g., burden), and are based on experiences of family members assisting cognitively impaired patients. Progress in the area of family care for elders can be enhanced through conceptualizing and measuring positive aspects of care for both members of the dyad and developing interventions to strengthen those aspects.

In a previous study using naturalistic inquiry, Sebern (1996) studied cognitively intact home health care patients and family members, assisting them to describe their care interactions used to prevent pressure ulcers. This study revealed that a theoretical basis for a 
care giving and receiving interaction was a construct called shared care. Although there are many possible components of interactions, the three components of shared care identified in the naturalistic inquiry were communication, decision making, and reciprocity (see Figure 1). These three components were defined as:

1. Communication (exchanging information about an illness experience)

2. Decision making (making care decisions)

3. Reciprocity (partnership, empathy, and listening when exchanging advice, aid, and emotional support)

The Shared Care Instrument (SCI) was developed to measure the construct. The purpose of this study was to assess the psychometric properties of the $\mathrm{SCl}$ including criterionrelated and construct validity.

\section{Background}

\section{Conceptual Framework}

Shared care is a dyadic process. Dyadic processes are based on the premise that each participant affects and is affected by the other (Gayle \& Preiss, 2002). The most obvious requirement for shared care is a chronically ill patient and a family member providing assistance. A family member assisting a patient is whomever the patient identifies; this person could be a relative or friend who is just like family.

Shared care interactions require cognitive ability to communicate, make decisions, and engage in reciprocal actions. In situations in which a patient has a severe cognitive deficit, other family members may substitute for the patient and engage in communication and decision making. However, researchers indicate that patients with mild to moderate cognitive impairment are able to answer questions about their own care and preferences with accuracy and reliability (Feinberg \& Whitlatch, 2001). When shared care occurs, both members of the dyad may experience positive outcomes. For example, when there is a high need for assistance and high shared care, both members of the dyad may experience benefits in their relationship; however, when there is a high need for assistance and low shared care, there can be negative effects on the relationship for both members of the dyad.

In a review of the caregiving literature, no one study investigated all three components of shared care; however, researchers did study components of shared care separately. For example, family researchers conceptualized communication and decision making as integral to family functioning (Thomas \& Olson, 1993). Other researchers found that communication 
(Pruchno, Burant, \& Peters, 1997a) and decision making (Deimling, Smerglia, \& Schaefer, 2001) affected caregiver outcomes such as mood, depression, and well-being. Pruchno and colleagues (1997a) found high agreement families had less burden, and suggested that high agreement families communicate more. Deimling and colleagues verified that higher levels of decision making satisfaction predicted lower levels of depression in family caregivers.

A number of studies were specifically relevant to the shared care construct in that reciprocity was studied. Researchers who studied reciprocity found that the opportunity to provide assistance and reciprocate within a family bolstered well-being for both members of the dyad (Archbold, Stewart, Greenlick, \& Harvath, 1992; Beach et al., 2000; Davey \& Eggebeen, 1998; Liang et al., 2001; Lyons, Zarit, Sayer, \& Whitlatch, 2002).

Both Sebern (1996) and Archbold and associates $(1990,1992)$ conceptualized reciprocity as an important component of family care relationships. Archbold and colleagues defined reciprocity as a giving and accepting of physical and emotional help with appreciation. These researchers conceptualized reciprocity as a component of mutuality. Mutuality was defined as an enduring quality of a relationship with four components: shared values, love, shared activities, and reciprocity. The Mutuality Scale was developed to measure the construct and its four components. Archbold and colleagues found that caregivers with low levels of mutuality experienced higher levels of caregiver role strain. In the current study we used the Mutuality Scale to test the criterion-related validity of the SCI. We expected significant correlations between shared care reciprocity and mutuality reciprocity.

In summary, several researchers found support for separate effects of communication, decision making, and reciprocity on outcomes for both patients and family members. Progress in this area could be enhanced through conceptualizing and measuring a construct that effectively captures the interactive effects of these three components.

\section{Procedures for Instrument Development}

Narrative data from the qualitative interviews conducted in the previous naturalistic inquiry were used to develop items to measure the three components of shared care (Sebern, 1996). Shared care communication was defined as exchanging information about an illness experience. Patients may under-report their experience to a family member, and family members may be uncertain about patient difficulties because they have inadequate information about the illness experience. Positive and negative statements were used to measure communication (e.g., "I have no one to talk to about how I am feeling."). Fifteen items were 
conceptualized as measuring communication; 7 were negatively worded and 8 positively worded. The communication items were the same for the patient and family member.

Shared care decision making was defined as making decisions to exert control over events and conditions in the patient's environment (e.g., "I decide when to call the doctor."). The patient's evaluation of the situation may be the basis for action, or the family member's understanding of the situation may be more important in making treatment decisions. The decision-making items were originally the same for the patient and family member. However, content experts suggested that the clarity of the decision-making items would be enhanced if the items focused only on the identified patient's decisionmaking capacity. In order to focus only on patient decision-making capacity, the pronouns Psychometric Evaluation of the Shared Care Instrument 177 for the decision-making items were changed in the family version of the SCI. The family member was asked to report their perception of the patient's decision-making capacity (e.g., "When my family member is not feeling well, s/he decides when to call the doctor."), and the patient was asked to report their own perception of their decision-making capacity (e.g., "When I am not feeling well, I decide when to call the doctor."). The 6 decision making items were different in the family version of the SCI in that they asked the family member to rate the patient's decision-making capacity.

Shared care reciprocity was defined as partnership, empathy, and listening when exchanging advice, aid, and emotional support (e.g., "We have a partnership."). Fifteen items measured reciprocity; 7 items were negatively worded (e.g., "I sacrifice my own needs.") and reverse coded. Reciprocity items were the same for the patient and family member. In the original instrument, 3 items measured satisfaction with decision making, reciprocity, and communication. These items were dropped from the scale based on feedback from content experts that satisfaction was not a good indicator of the shared care components.

Content validity of the SCI was supported by a panel of nurse experts who were given specific definitions for the components of shared care and asked to judge how relevant the items were to the conceptual definitions. There was $100 \%$ agreement among the experts about relevancy of items to communication and decision making, and $80 \%$ agreement about relevancy of reciprocity items. The experts reported that there was a lack of clarity in the decision-making items, because they were not sure if the items related to the patient's or caregiver's decisionmaking capacity. The instrument was revised based on recommendations from the content experts. These revisions focused 6 decision-making items on the identified patient's decisionmaking capacity, and removed the 3 satisfaction items. The original SCI had 36 items. As mentioned above, the decision-making items differed because the family member rated the

\section{Sebern 4}


patient's decision-making capacity, but the patient rated their own decision-making capacity. The reciprocity and communication items were the same on the patient and family member versions in that they each rated their own level of communication and reciprocity in the relationship.

Each member of the dyad was conceptualized as having a unique experience in the relationship because of their role as either a patient or family member providing assistance. Because it was important to measure their unique experience of shared care, the patient and family member completed separate surveys.

A pilot test was conducted to evaluate the study's methods. Packages of instruments were mailed to 10 home health care family dyads. Out of 10 surveys mailed to patient-caregiver dyads, $70 \%$ of patients and $80 \%$ of caregivers returned completed surveys. The participants who returned their completed surveys indicated that family dyads were able to understand the written directions, complete, and return the mailed surveys in accordance with the study's methods.

\section{Description, Administration, and Scoring of the Instrument}

The original SCI had a total of 36 items; 15 communication items, 6 decision-making items, and 15 reciprocity items. The 13 negatively worded items (6 communication and 7 reciprocity) were reverse scored so that higher scores indicated higher shared care. The SCI asked participants to rate their agreement with items on a 6-point Likert-type scale ranging from strongly disagree (1) to strongly agree (6). After reverse coding negatively worded items, subscales were summarized into a subscale score, and higher scores reflected better communication, decision making, and reciprocity for each member of the dyad.

We used the Simplified Measure of Gobbledegoop (SMOG) Readability Formula to analyze the SCI readability (U.S. Department of Health and Human Services, 1989). Based on the SMOG formula, the SCI is at a seventh grade reading level. The focus of analysis was to determine if empirical data supported shared care as originally conceptualized with three components.

\section{Methods}

\section{Design, Sample, and Procedures}

After Institutional Review Board approval from university and health care organizations, a convenience sample was obtained. Two home health care agencies serving both urban and rural populations in a Midwestern state participated. Personnel from the home health care 
agencies' information system departments selected patients from their databases who met study criteria. Criteria for inclusion were: over 18 years of age, cognitive ability and vision intact, and living with a person who provided unpaid assistance.

Two hundred thirty-five survey packages were mailed to the home health care family dyads. Each survey package included a patient version and a family member version of the SCI (36 items), demographic questions, a self-addressed stamped return envelope, and written instructions. To assess criterion-related validity of the SCl, we included the Mutuality Scale (15 items) in 100 packages. The rationale for including only 100 Mutuality scales was to reduce burden on participants by only collecting data needed to test the correlation between mutuality and shared care. Participants were asked not to talk to the other member of the dyad about their answers until they completed the survey. Both members were encouraged to participate. Since all participating patients had a person providing them with assistance (eligibility criteria), if only one member of the dyad decided to participate their survey was included in data analysis.

To support dyad participation a pre-notification letter was mailed prior to mailing the survey package, a $\$ 2.00$ token of appreciation was clipped to each survey, and a postcard reminder was sent 1 week after the survey. At 4 weeks a second survey was sent to nonresponders, and at 6 weeks non-responders received a follow-up phone call to inquire about the survey. The response rate was about $48 \%$ for both groups. In the patient group, 116 returned surveys and 20 patients returned their surveys without family member participation. In the family member group, 117 returned surveys, and 21 of the family members participated without patient participation. About $8 \%$ of patients $(n=19)$ did not participate due to death or institutionalization. Other reasons reported for not participating were language differences, too busy, and wrong address.

\section{Instruments}

The primary construct of interest in this study was shared care; however, to evaluate validity of the SCI, the Mutuality Scale was administered. Archbold and associates (1992) defined mutuality as an enduring quality in a relationship consisting of love, shared pleasurable activities, shared values, and reciprocity. There are four subscales in the Mutuality Scale that measured each of these components. Two items measured shared values; an example of a shared values item was, "To what extent do you see eye to eye?" Three items measured love; an example of a love item was, "How close do you feel to him or her?" Four items measured shared pleasurable activities; an example of one of these items was, "How much do you enjoy sharing past experiences with him or her?" Six items measured Psychometric Evaluation of the 
Shared Care Instrument 179 reciprocity; an example of one of these items was, "How much does he or she express feelings of appreciation for you and the things you do?" The 15-item Mutuality Scale asks participants to rate their agreement with statements on a 5-point Likerttype scale, ranging from "not at all" (0) to "a great deal" (4). Scores can range from 0 to 60 with higher scores indicating higher mutuality. In the current study, Cronbach's alpha for the Mutuality Scale for both patient and the family member groups was .95. Because both shared care and mutuality included reciprocity scales, a strong correlation between the reciprocity subscales of the two instruments would support criterion-related validity of the $\mathrm{SCI}$ reciprocity subscale.

\section{Statistical Methods}

Methods for estimating internal consistency (Cronbach's alpha and inter-item correlations) and homogeneity (item-total scale correlations) were used to test the reliability of the $\mathrm{SCl}$ and subscales. Validity of the $\mathrm{SCl}$ was tested with factor analysis, paired $t$ tests, and by examining correlations between the $\mathrm{SCl}$ and Mutuality Scale. Patients and family members were conceptualized as having different perspectives of their shared experience. We analyzed patient and family data separately for conceptual reasons, because of the small sample, and due to measurement error associated with dependency in dyad data. When identical measures are used for each dyad member the within-dyad correlation can be overestimated because of correlated measurement error (Cook, 1994). A correlated measurement error is an association between two items beyond that due to the correlation between their respective latent variables. For example, reciprocity may be influenced by a similarity between patient's and family member's personalities. Correlated errors can occur with any two latent variables, but they are especially likely when parallel item sets are used to measure a construct in two members of a dyad. Correlated error may be due to item content, specific wording, or methodological factors. The overestimation of the within-dyad correlation will lead to an underestimation of the unique (partial) relationships. To avoid a problem with correlated measurement error in dyad data, we analyzed patient and family groups separately. In addition, the data of patients and caregivers should not be combined because they are also two separate populations.

The Statistical Package for the Social Sciences (1999) subprograms Frequencies, Reliability, and Factor were used to compute sample demographic analysis and instrument psychometric properties. Incomplete and missing data were handled by examining for patterns in the missing data and imputing missing data using a multiple imputation probability model (Schafer, 1997). Pearson's correlation was used to examine the association between Mutuality 
subscales and shared care subscales. To test for a difference between patient and family member shared care subscale means, a two-tailed paired $t$ test was used. We were interested in the difference between patient and family member shared care, because researchers reported that different perceptions of the caregiving situation contribute to relationship strain (Horowitz, Goodman, \& Reinhardt, 2004; Lyons et al., 2002).

\section{Results}

Demographic characteristics of the patient and family members are displayed in Table 1. Patients' ages ranged from 28 to 92 years (mean was 70 years, SD 15 years). Fifty-six percent of the patients were female. A large percent of patients were White (94\%), 4\% were Black, and less than 1\% were American Indian. The most frequent patient medical conditions were cardiac disease and diabetes. The remaining medical conditions included neoplasm, paraplegia, chronic lung disease, digestive system problems, and musculoskeletal problems.

The age of family members ranged from 16 to 90 years (mean was 63 years, SD 17 years). Sixty-four percent of family members assisting the patients were female. A good number of family members were White (95\%), less than $3 \%$ were Black, and $2 \%$ were American Indian. Hispanic ethnicity was reported by $3 \%$ of patients and $2 \%$ of family members. A small number of participants had completed college; however, most had completed high school.

Some questions asked participants about their role and length of relationship with the other member of the dyad. Descriptive statistics were used to evaluate these responses. In patients who identified their relationship to a family member assisting them, $66 \%$ of patients were related to a family member as spouse or partner, $19 \%$ of patients were parents, $3 \%$ were children, and $4 \%$ of patients were related as friends or siblings. The dyads had known each other on average for 43 years. Forty percent of the patients and $36 \%$ of family members reported that they were both a caregiver and recipient. Participants who viewed themselves as givers and recipients of care may suggest that these participants exchanged substantial assistance to each other.

\section{Missing Data}

The study's response rate was $48 \%$ with 116 patients and 117 family members participating. The analysis included evaluating for patterns of missing data. Most missing data was due to skipped pages or randomly omitted answers. Cases were deleted if more than $15 \%$ (i.e., 6) of scale items were missing. Six patient cases and 8 family member cases were deleted due to missing more than $15 \%$ of data. Using the remaining case, patterns of missing data were 
analyzed and none of the items were missing more than three data points. Multiple imputation methods impute missing data by imposing a probability model on the complete data (observed and missing values) (Schafer, 1997). The final sample size was 110 patients and 109 family members.

\section{Factor Analysis}

Several factor analysis models were used, specifying orthogonal (varimax) and oblique (promax) rotations, to explore the nature and underlying dimensions of the $\mathrm{SCl}$ in each group. The results of maximum likelihood factor analysis with oblique rotation resulted in the best model. Maximum likelihood factor analysis estimates the population correlation matrix, acquired from the matrix obtained from the actual data. Maximum likelihood is preferred for factor extraction if the goal is to identify a theoretical solution based on underlying constructs that produce scores in the observed variables (Tabachnick \& Fidell, 2001).

A four-factor solution for both groups was identified using maximum likelihood factor analysis. Although a three-factor structure was hypothesized, it is not unusual to identify additional factors in an exploratory factor analysis. Criteria used to identify the number of factors were eigenvalues greater than 1 , location of a distinct break in the slope of the scree plot, more than $5 \%$ variance explained by a factor, and interpretability of factors (Pett, Lackey, \& Sullivan, 2003).

Three factors were consistent with the components of shared care, but the fourth was not. The fourth factor contained only negatively worded items that were originally conceptualized as indicators of low levels of reciprocity and communication. For example, negative items loading on the fourth factor were "I get no reward." and "We usually don't agree about what ought to be done when one of us is ill." The same six items loaded above .30 on the fourth factor in each group. Once this factor and its items were identified, the factor was reviewed by a panel of nurse researchers in order to name the factor. These nurse researchers suggested naming the factor balance, because the factor pointed to a lack of balance between providing and receiving assistance. This factor was not consistent with the model of shared care. It was not initially clear whether the factor should be removed from the $\mathrm{SCl}$, so it was retained in the analysis.

A meaningful comparison between patients' and family members' shared care required a stable factor structure across both groups. To identify a stable factor structure, we did a preliminary exploration and compared factor structures between patient and family groups. The following statistical and conceptual criteria were used to compare the patient and family factor 
structures: (a) item factor loading was greater than .30, (b) items did not load on different factors between groups, and (c) interpretability of factors. With our preliminary exploration, 4 items in the patient structure, and 1 item in the family structure did not load on any factor. In addition, 5 items loaded on different factors for each group. Thus a total of 10 items were removed because they were not unique to one factor or their factor loading was below .30. The preliminary analysis led to the retention of 26 items whose properties were tested in the final step of the factor analysis. Factor analysis was performed with the 26 -item SCI to determine the nature of factors that could explain the correlations among the responses.

Maximum likelihood factor analysis specifying four factors and using promax (oblique) and varimax (orthogonal) rotations supported a model that represented data adequately for both groups. Use of varimax and promax rotations did not reveal substantially different results. A solution using oblique rotation was selected because communication, decision making, and reciprocity were conceptualized as interrelated concepts.

\section{Item Analysis and Descriptive Statistics}

Descriptive statistics, including item means, standard deviations, inter-item correlations, and item-total correlations were computed and examined. Descriptive statistics, item-total score correlations, and alpha coefficients for the four SCI subscales appear in Table 2. On a 6-point scale $(1=$ completely disagree to $6=$ completely agree), the means ranged from 3.9 (family communication) to 4.9 (patient decision making). Examination of the item-to-total correlations indicated that all items positively correlated with their respective subscale total score. For example, the patient reciprocity subscale item-to-total correlations ranged from .20 to .67, and ranges for patient communication were .51 to .73. This range of item-to-total correlations was considered to be acceptable (Nunnally \& Bernstein, 1994).

\section{Reliability Estimates}

Estimates of internal consistency reliability in both groups for the four subscales were found to have adequate reliability for exploratory research. The internal consistency of the four subscales was not improved substantially by deleting any of the items. Cronbach's alpha for the subscales ranged from .77 (family communication and balance) to .84 (patient balance).

\section{Construct Validity of the SCI}

Bartlett's test of sphericity and KMO measure of sampling adequacy were calculated to evaluate the strength of linear association among items. Bartlett's test was significant for the 
patient group $\left(\mathrm{x}^{2}=1251, d f=325, p=.000\right)$ and for the family group $\left(\mathrm{x}^{2}=1152, d f=325, p\right.$ $=.000)$. The KMO statistic for the patient group was .75 , and .70 for the family group. The KMO statistic of .60 or higher indicates that a correlation matrix is factorable (Pett et al., 2003).

Four factors were extracted from the patient and family member data using maximum likelihood factor analysis with promax rotations. An examination of the goodness of fit chisquare ratio revealed that a four-factor model represented the data adequately and the chisquare was significant $(p=.000)$. Because of difficulties in using chi-square as a measure of goodness of fit, Carmines and Mclver (1981) recommended a chi-square degrees of freedom ratio of less than 3 to 1, as indicative of an acceptable fit between a hypothetical model and sample data. The goodness of fit chi-square ratio for both patient and family SCI factor model was 1.4 to 1 , supporting an acceptable fit between the model and data.

The four-factor extracted solution for each group was analyzed statistically and conceptually using the following criteria: (a) items load on one factor (>.30), (b) items do not load substantively on two or more factors (>.50), (c) items load on the same factor in both groups, and (d) interpretability of factors. An analysis of the factor loadings in the patient group indicated that all 26 items loaded as conceptualized on the patient factors reciprocity, balance, decision making, and communication. (See Appendix A, Patient Pattern Matrix.) In both groups the first factor was reciprocity and the fourth was communication. Decision making was the second factor for the family group and the third factor for the patient group. Names for the factors identified in this factor analysis were derived from the construct shared care, which was identified in a previous naturalistic inquiry. Factor analysis supported the original conceptualization of three factors; communication, decision making, and reciprocity; in both groups. As mentioned previously, a new factor was identified and labeled balance. Balance items were defined by nurse researchers as indicators of a lack of balance between providing and receiving assistance (e.g., "I sacrifice my own needs when s/he is sick.").

The reciprocity factor indicated empathy, listening, and partnership in managing the illness. For example, one reciprocity item indicated that the dyad tried out different strategies to manage an illness (e.g., "When we try something that does not make my family member feel better we will try something else."). The decision-making factor suggested the patient's capacity for making decisions about care (e.g., "When I am not feeling well, I decide when to call the doctor."). As mentioned previously, the family member rates a patient's decision-making capacity (e.g., "When my family member is not feeling well, s/he decides when to call the doctor."). The communication factor implied that a patient and family member talked with each other and asked for advice. All communication items are negatively worded statements and 
were reverse coded. An example of a communication item was, "There is no one to talk to about how I am feeling."

Although all items loaded on the patient factors as conceptualized, for the family members three items did not load as expected. (See Appendix B, Family Pattern Matrix.) One balance item (i.e., "My family member complains about feeling sick when there is nothing wrong.") had factor loading of .27 on balance and .29 factor loading on reciprocity. Also, one decision-making and one reciprocity item loaded with the balance factor. This decision- making item (i.e., "When my family member is sick s/he does as much as $\mathrm{s} / \mathrm{he}$ can for himself.") had a factor loading of .30 on the family decision-making factor and .39 on the family balance factor. The reciprocity item that loaded on balance was, "If my family member is uncomfortable I know it, s/he will tell me what $s /$ he wants." These three items were retained on the factors as originally conceptualized in order to maintain congruency between patient and family member factor structures.

Correlations between the four factors for the patient and family member are presented in Table $3 \mathrm{SCI}$ Factor Score Intercorrelation Matrix (26 Items). Correlations between the factors were relatively low (family factors $r=.10$ to .35 ; patient factors $r=-.16$ to .37 ), supporting the finding of the factor analysis that the $\mathrm{SCl}$ measured distinct aspects of shared care. In the patient factor structure there was a negative correlation between balance and decision making, and small correlations with reciprocity (.01) suggesting that balance may not be a component of patient shared care. Using maximum likelihood factor analysis, a four-factor solution (communication, decision making, reciprocity, balance) explained $53.4 \%$ of variance in the patient data, and $49.4 \%$ of variance in the family member data. Table 4 shows the variance explained by the four-factor solution.

To determine if there was a significant difference in subscale means between patient and family members, a two-tailed paired $t$ test was used. Significant differences were found between patient and family members on all shared care subscales. The family member had significantly lower scores on communication and decision-making subscales, suggesting that family members perceived less communication and decision making in their relationship compared to what patients perceived. However, family member means were higher for the reciprocity subscale, suggesting that family members perceived more reciprocity in the relationship than the patient (see Table 5). 


\section{Criterion-Related Validity}

Criterion validity of the $\mathrm{SCl}$ was evaluated by examining correlations between $\mathrm{SCI}$ factors and Archbold and Stewart's mutuality factors (Archbold et al., 1992). The Mutuality Scale measured four factors (love, reciprocity, shared values, and activities). It was hypothesized that the Mutuality Scale's reciprocity subscale would positively correlate with the SCI reciprocity subscale. We also examined the correlations between the other subscales.

The SCI reciprocity factor correlated in the expected positive direction with all the mutuality factors for both groups. In the patient group, SCI reciprocity correlated with all the mutuality subscales $(r=.40$ to $.59, p<.01$ ) (see Table 6$)$. This finding suggested that patient reciprocity scores are associated with the Mutuality Scale's reciprocity, love, shared values and activities subscales.

In the family members the $\mathrm{SCI}$ factors reciprocity and decision making correlated with all the mutuality factors $(r=.31$ to $.60, p<.01)$. In addition, family shared care communication and balance factors had significant correlations with mutuality reciprocity and shared activity factors ( $r=.24$ to $.38, p<.05)$. These findings support the criterion-related validity of the SCI because of the significant positive association between reciprocity subscales and mutuality subscales in both groups, and the additional associations of family decision-making, communication, and balance subscales with mutuality subscales.

\section{Discussion}

Cronbach's alpha, an estimate of internal consistency, was acceptable for a new instrument for both patients and family members. In addition, the four SCI factors were found to have adequate reliability in both groups for exploratory research. The entire SCI may not be the best reflection of reliability due to the following reasons. First, there is a need to further clarify whether the balance factor is or is not a component of shared care. And secondly, because shared care is a multidimensional construct, it is important to evaluate the psychometrics of the four subscales individually.

Exploratory factor analysis was used to summarize the interrelationships among items on the SCl. The factor analysis supported the original conceptualization of three components of shared care (communication, reciprocity, decision making), and a new factor, balance, was identified. Reciprocity was the factor that explained most of the variance in shared care for both groups. The nature of the reciprocity factor suggested that partnership, empathy, and listening were attributes of exchanging assistance. Only negatively worded items loaded on balance and communication factors, the negative items were originally conceptualized as indicators of low 
reciprocity and communication. All items loading on reciprocity or decision-making factors were positively worded. The separation of negative and positive items on factors suggested that the SCl's positive and negative dimensions are separate phenomena.

Decision making and communication were identified by researchers as important components of family functioning and predictors of caregiver outcomes (Deimling et al., 2001; Pruchno, Burant, \& Peters, 1997b). When a family experiences a chronic illness, longstanding communication and decision-making patterns may be challenged due to lack of knowledge and skills related to the new experience. Family dyads may benefit when provided with opportunities to learn new skills, such as how to interpret and respond to symptoms, communicate care preferences, and make care-related decisions.

Results from a paired $t$ test indicated significant differences between patient and family perceptions on the SCI. The significant differences between the group means suggested that the $\mathrm{SCl}$ can discriminate between different levels of shared care. Researchers reported that discrepancies between a patient and family member about their shared experience contributes to poor outcomes for both members of the dyad (Horowitz et al., 2004; Lyons et al., 2002; Pruchno et al., 1997b). Further research is required to determine how differences in patient and family views of shared care affect their outcomes.

The data from this study supported the $\mathrm{SCl}$ as a multidimensional tool. Researchers have previously conceptualized communication, reciprocity, and decision-making separately; however, the family care literature may be advanced by conceptualizing and measuring a construct that effectively captures the interactive effects of these components. The revised SCI is currently being tested to confirm its factor structure.

\section{Limitations}

One limitation of this study was its exploratory nature and small sample ( $n=110$ patients, and $n=109$ family members). Also, the sample was primarily from a Midwestern state, White, elderly with chronic physical illnesses, and receiving home health care services, and dyads knew each other for a long time. Additional use and testing of the $\mathrm{SCl}$ is needed to determine if relationships exist among the dimensions of shared care across age, ethnicity, persons with mental illness, and in short-term relationships.

Well-defined concepts with conceptual and construct validity enhance communication among scholars. The concepts of communication, decision making, reciprocity, and balance received empirical support by this exploratory factor analysis. In addition, criterion validity was supported by significant correlations between the $\mathrm{SCI}$ subscales and mutuality subscales. A 
larger database is needed to confirm the factor structure of $\mathrm{SCl}$. Relationships between levels of shared care and outcomes for both members of the dyad should be established based on theory and hypothesis testing. Further research should explore the effects of differences between levels and directions of shared care on outcomes for both members of the dyad.

\section{Conclusions}

The purpose of this study was to evaluate selected psychometric properties of the $\mathrm{SCl}$ as well as its construct and criterion validity. Reliability, construct, and criterion-related validity findings from this study support continued testing of the $\mathrm{SCl}$. Based on the findings from this study, the $\mathrm{SCl}$ was revised and Shared Care Instrument-Revised (SCI-R) is currently being tested to confirm the factor structure with a new sample of home health care family dyads. A psychometrically sound measure of shared care may contribute to progress in the area of family caregiving through the creation, refinement, and measurement of a construct that captures effectively the interactive effect of family care. The measurement of shared care offers scholars a way to study care interactions inclusive of patients and family members, assist with identifying areas of difficulty, and guide interventions to assist in areas of difficulty.

\section{References}

Archbold, P. G., Stewart, B. J., Greenlick, M. R., \& Harvath, T. A. (1990). Mutuality and preparedness as predictors of caregiver role status. Research in Nursing and Health, 13, 375-384.

Archbold, P. G., Stewart, B. J., Greenlick, M. R., \& Harvath, T. A. (1992). The clinical assessment of mutuality and preparedness in family caregivers to frail older people. In S. G. Funk, E. M. Tornquist, M. T. Champagne, \& L. A. Copp (Eds.), Key aspects of elder care (pp. 328-339). New York: Springer.

Beach, S. R., Schulz, R., Yee, J. L., \& Jackson, S. (2000). Negative and positive health effects of caring for a disabled spouse: Longitudinal findings from the caregiver health effects study. Psychology \& Aging, 15(2), 259-271.

Carmines, E. G., \& Mclver, J. P. (1981). Analyzing models with unobserved variables: Analysis of covariance structures. Social measurement: Current Issues, 65-115.

Cook, W. L. (1994). A structural equation model of dyadic relationships within the family system. Journal of Consulting and Clinical Psychology, 62(3), 500-509. 
Davey, A., \& Eggebeen, D. J. (1998). Patterns of intergenerational exchange and mental health. Journals of Gerontology Series B--Psychological Sciences \& Social Sciences, 53(2), $86-95$.

Deimling, G. T., Smerglia, V. L., \& Schaefer, M. L. (2001). The impact of family environment and decision-making satisfaction on caregiver depression: A path analytic model. Journal of Aging \& Health, 13(1), 47-71.

Feinberg, L. F., \& Whitlatch, C. J. (2001). Are persons with cognitive impairment able to state consistent choices? Gerontologist, 41(3), 374-382.

Gayle, B. M., \& Preiss, R. W. (2002). An overview of dyadic processes in interpersonal communication. In M. Allen, R. W. Preiss, B. M. Gayle, \& N. A. Burrell (Eds.), Interpersonal communication research: Advances through meta-analysis (pp. 111-124). Mahwah, NJ: Lawrence Erlbaum Associates.

Horowitz, A., Goodman, C., \& Reinhardt, J. (2004). Congruence between disabled elders and their primary caregivers. The Gerontologist, 44(4), 532-542.

Liang, J., Krause, N. M., \& Bennett, J. M. (2001). Social exchange and well-being: Is giving better than receiving? Psychology and Aging, 16(3), 511-523.

Lyons, K. S., Zarit, S. H., Sayer, A. G., \& Whitlatch, C. J. (2002). Caregiving as a dyadic process: Perspectives from caregiver and receiver. Journals of Gerontology Series BPsychological Sciences and Social Sciences, 57(3), 195-204.

Nunnally, J. M., \& Bernstein, I. R. (1994). Psychometric theory (3rd ed.). New York: McGraw-Hill Series in Psychology.

Pett, M. A., Lackey, N. R., \& Sullivan, J. J. (2003). Making sense of factor analysis. The use of factor analysis for instrument development in health care research. Thousand Oaks, CA: Sage Publications.

Pruchno, R. A., Burant, C. J., \& Peters, N. D. (1997a). Typologies of caregiving families: Family congruence and individual well-being. The Gerontologist, 37(2), 157-167.

Pruchno, R. A., Burant, C. J., \& Peters, N. D. (1997b). Coping strategies of people living in multigenerational households: Effects on well-being. Psychology \& Aging, 12(1), 115124.

Reinardy, J. R., Kane, R. A., Huck, S., Call, K. T., \& Shen, C. T. (1999). Beyond burden: Two ways of looking at caregiving burden. Research on Aging, 21(1), 106-127.

Schafer, J. L. (1997). Analysis of incomplete multivariate data. London: Chapman \& Hall.

Sebern, M. (1996). Explication of the construct of shared care and the prevention of pressure sores in home health care. Research in Nursing and Health, 19, 183-192. 
Statistical Package for the Social Sciences (SPSS). (1999). SPSS Base (Version 9.0). Chicago: SPSS.

Tabachnick, B. G., \& Fidell, L. S. (2001). Using multivariate statistics (4th ed.). Boston: Allyn and Bacon.

Thomas, V., \& Olson, D. H. (1993). Problem families and the Circumplex Model: Observational assessment using the Clinical Rating Scale (CRS). Journal of Marital and Family Therapy, 19(2), 159-175.

U.S. Department of Health and Human Services. (1989). Making health communication programs work. Washington, DC: National Cancer Institute.

\section{Acknowledgments}

DHHS PHS NIH NINR provided financial support for this research. Grant number: 5T32 NR07102-03. Title of award: Patient Centered Informational Interventions PI: Sandra Ward DHHS PHS NIH NINR.

\section{Offprints}

Requests for offprints should be directed to Margaret D. Sebern, PhD, RN, University of Wisconsin-Milwaukee, College of Nursing, Cunningham Hall, Rm. 421, PO Box 413, Milwaukee, WI 53201. E-mail: marge@sebern.com 


\section{Appendix}

\section{Appendix A. Patient SCI Pattern Matrix (26 Items)}

\begin{tabular}{|c|c|c|c|c|}
\hline & \multicolumn{4}{|c|}{ Factor } \\
\hline & Reciprocity & Balance & Decision Making & Communication \\
\hline 15 Reciprocity & .874 & & & \\
\hline 16 Reciprocity & .733 & & & \\
\hline 17 Reciprocity & .666 & & & \\
\hline 29 Reciprocity & .535 & & & \\
\hline 18 Reciprocity & .460 & & & \\
\hline 24 Reciprocity & .430 & & & \\
\hline 25 Reciprocity & .426 & & & \\
\hline 14 Reciprocity & .415 & & & \\
\hline 33 Reciprocity & .356 & & & \\
\hline 32 Reciprocity & .325 & & & \\
\hline 12 Balance & & .878 & & \\
\hline 10 Balance & & .729 & & \\
\hline 9 Balance & & .693 & & \\
\hline 11 Balance & & .669 & & \\
\hline 13 Balance & & .626 & & \\
\hline 5 Balance & & .474 & & \\
\hline 21 Decision making & & & .787 & \\
\hline 22 Decision making & & & .737 & \\
\hline 20 Decision making & & & .652 & \\
\hline 19 Decision making & & & .574 & \\
\hline 30 Decision making & & & .436 & \\
\hline 23 Decision making & & & .422 & \\
\hline 2 Communication & & & & .864 \\
\hline 1 Communication & & & & .761 \\
\hline 3 Communication & & & & .655 \\
\hline 4 Communication & & & & .583 \\
\hline
\end{tabular}

Note. Extraction method: Maximum likelihood. Rotation method: Proxmax with Kaiser normalization. A rotation converged in 6 iterations. 


\section{Appendix B. Family Member SCI Pattern Matrix (26 Items)}

\begin{tabular}{|c|c|c|c|c|}
\hline & \multicolumn{4}{|c|}{ Factor } \\
\hline & Reciprocity & Balance & Decision Making & Communication \\
\hline 24 Reciprocity & .743 & & & \\
\hline 17 Reciprocity & .656 & & & \\
\hline 18 Reciprocity & .634 & & & \\
\hline 25 Reciprocity & .590 & & & \\
\hline 33 Reciprocity & .539 & & & \\
\hline 16 Reciprocity & .453 & & & \\
\hline 14 Reciprocity & .394 & & & \\
\hline 29 Reciprocity & .390 & & & \\
\hline 32 Reciprocity & .382 & & & \\
\hline 21 Decision making & & .876 & & \\
\hline 23 Decision making & & .829 & & \\
\hline 22 Decision making & & .704 & & \\
\hline 19 Decision making & & .575 & & \\
\hline 20 Decision making & & .338 & & \\
\hline 5 Balance & .295 & & .269 & \\
\hline 10 Balance & & & .885 & \\
\hline 12 Balance & & & .643 & \\
\hline 9 Balance & & & .624 & \\
\hline 11 Balance & & & .507 & \\
\hline 15 Reciprocity & & & -.447 & \\
\hline 30 Decision making & & .293 & .402 & \\
\hline 13 Balance & & & .306 & \\
\hline 3 Communication & & & & .926 \\
\hline 2 Communication & & & & .829 \\
\hline 1 Communication & & & & .501 \\
\hline 4 Communication & & & & .423 \\
\hline
\end{tabular}

Note. Extraction method: Maximum likelihood. Rotation method: Proxmax with Kaiser normalization. A rotation converged in 6 iterations. 


\section{Table 1: Demographic Characteristics of Patients and Family Member}

Participants

\begin{tabular}{|c|c|c|}
\hline Demographic Item & Patients $(n=110)$ & Family Members $(n=109)$ \\
\hline Age & $70 \pm 15$ years & $63 \pm 17$ years \\
\hline \multicolumn{3}{|l|}{ Gender } \\
\hline Female & $56 \%(64)$ & $64 \%(70)$ \\
\hline \multicolumn{3}{|l|}{ Relationship } \\
\hline Spouse & $66 \%(72)$ & $64 \%(70)$ \\
\hline Parent & $19 \%(21)$ & $3 \%(4)$ \\
\hline Child & $3 \%(4)$ & $24 \%(26)$ \\
\hline Friend/sibling & $4 \%(5)$ & $6 \%(6)$ \\
\hline Other & $8 \%(8)$ & $3 \%(3)$ \\
\hline \multicolumn{3}{|l|}{ Race } \\
\hline White & $94 \%(103)$ & $95 \%(103)$ \\
\hline Black & $4 \%(5)$ & $3 \%(4)$ \\
\hline American Indian & $<1 \%$ (1) & $2 \%(2)$ \\
\hline Other & $<1 \%$ (1) & $0 \%$ \\
\hline \multicolumn{3}{|l|}{ Ethnicity } \\
\hline Hispanic & $3 \%(3)$ & $2 \%(2)$ \\
\hline \multicolumn{3}{|l|}{ Education } \\
\hline $\begin{array}{l}\text { College degree/post- } \\
\text { college }\end{array}$ & $20 \%(23)$ & $25 \%(28)$ \\
\hline High school diploma & $57 \%(63)$ & $55 \%(60)$ \\
\hline Less than high school & $22 \%(24)$ & $19 \%(21)$ \\
\hline \multicolumn{3}{|l|}{ Medical diagnosis } \\
\hline Circulatory & $48 \%(51)$ & \\
\hline Diabetes & $14 \%(16)$ & \\
\hline Paraplegia & $10 \%(12)$ & \\
\hline Neoplasm & $10 \%(12)$ & \\
\hline Chronic lung & $6 \%(7)$ & \\
\hline Musculoskeletal & $4 \%(4)$ & \\
\hline Digestive system & $3 \%(3)$ & \\
\hline Other & $5 \%(5)$ & \\
\hline
\end{tabular}




\section{Table 2: Descriptive and Psychometric Statistics for SCI Total and Subscale Scores (26 Items)}

\begin{tabular}{|c|c|c|c|c|}
\hline Factor/Scale & $\begin{array}{l}\text { Scale Item } \\
\text { Means }\end{array}$ & $\begin{array}{l}\text { Subscale/Scale } \\
\text { Means }(S D)\end{array}$ & $\begin{array}{l}\text { Item-to-Total Score } \\
\text { Correlation, Ranges }\end{array}$ & Cronbach's a \\
\hline \multicolumn{5}{|l|}{ Reciprocity } \\
\hline No. of items 10 & $\mathrm{PT}=4.6$ & $\mathrm{PT}=46.8(8.4)$ & $\mathrm{PT}=.20-.67$ & $\mathrm{PT}=.78$ \\
\hline Score range $10-60$ & $\mathrm{FM}=4.8$ & $\mathrm{FM}=48.0(7.2)$ & $\mathrm{FM}=.22-.57$ & $\mathrm{FM}=.75$ \\
\hline \multicolumn{5}{|l|}{ Decision making } \\
\hline No. of items 6 & $\mathrm{PT}=4.9$ & $\mathrm{PT}=29.7$ (5.3) & $\mathrm{PT}=.41-.69$ & $\mathrm{PT}=.78$ \\
\hline Score range 6-36 & $\mathrm{FM}=4.5$ & $F M=27.2(6.2)$ & $\mathrm{FM}=.32-.72$ & $\mathrm{FM}=.79$ \\
\hline \multicolumn{5}{|l|}{ Communication } \\
\hline No. of items 4 & $\mathrm{PT}=4.2$ & $\mathrm{PT}=16.8(5.8)$ & $\mathrm{PT}=.51-.73$ & $\mathrm{PT}=.81$ \\
\hline Score range 4-24 & $\mathrm{FM}=3.9$ & $\mathrm{FM}=15.8(5.3)$ & $\mathrm{FM}=.35-.70$ & $\mathrm{FM}=.77$ \\
\hline \multicolumn{5}{|l|}{ Balance } \\
\hline No. of items 6 & $\mathrm{PT}=4.5$ & $\mathrm{PT}=36.4$ (8.9) & $\mathrm{PT}=.28-.62$ & $\mathrm{PT}=.84$ \\
\hline Score range 6-36 & $\mathrm{FM}=4.1$ & $F M=32.9(8.1)$ & $\mathrm{FM}=.32-.57$ & $\mathrm{FM}=.77$ \\
\hline \multicolumn{5}{|l|}{ SCI total score } \\
\hline No. of items 26 & $\mathrm{PT}=4.6$ & $\mathrm{PT}=121(16)$ & $\mathrm{PT}=(-.01)-.51$ & $\mathrm{PT}=.80$ \\
\hline Score range 26-156 & $\mathrm{FM}=4.4$ & $\mathrm{FM}=115(16)$ & $F M=(-.01)-.56$ & $\mathrm{FM}=.82$ \\
\hline
\end{tabular}

Note. Individual item responses 1-6. PT = patient $(n=110) ; \mathrm{FM}=$ family member $(n=109)$. 
Table 3: SCI Factor Score Intercorrelation Matrix (26 Item)

\begin{tabular}{lccc}
\hline Factor & Reciprocity & Decision Making & Communication \\
\hline Decision making & $\mathrm{PT}=.37$ & & \\
& $\mathrm{FM}=.31$ & $\mathrm{PT}=.03$ & \\
Communication & $\mathrm{PT}=.18$ & $\mathrm{FM}=.35$ & \\
Balance & $\mathrm{FM}=.27$ & $\mathrm{PT}=-.16$ & $\mathrm{PT}=.26$ \\
& $\mathrm{PT}=.01$ & $\mathrm{FM}=.15$ & $\mathrm{FM}=.35$ \\
\hline
\end{tabular}

Note. Extraction method: Maximum likelihood. Rotation method: Promax with Kaiser normalization. Patient factors are correlated with other patient factors, and family member factors are correlated with other family member factors. PT = patient $(n=110) ; \mathrm{FM}=$ family member $(n=109)$. 
Table 4: Variance Explained by the Four SCI Extracted Factors (26 Item)

\begin{tabular}{lccc}
\hline & Eigenvalue & \% Variance & Cumulative $\%$ \\
\hline Reciprocity & $\mathrm{PT}=5.0$ & $\mathrm{PT}=19.5 \%$ & $\mathrm{PT}=19.5 \%$ \\
Balance & $\mathrm{FM}=5.3$ & $\mathrm{FM}=20.7 \%$ & $\mathrm{FM}=20.7 \%$ \\
& $\mathrm{PT}=4.4$ & $\mathrm{PT}=17.0 \%$ & $\mathrm{PT}=36.5 \%$ \\
Decision making & $\mathrm{FM}=2.4$ & $\mathrm{FM}=9.2$ & $\mathrm{FM}=42.5 \%$ \\
& $\mathrm{PT}=2.3$ & $\mathrm{PT}=8.8 \%$ & $\mathrm{PT}=45.3 \%$ \\
Communication & $\mathrm{FM}=3.2$ & $\mathrm{FM}=12.5 \%$ & $\mathrm{FM}=33.2 \%$ \\
& $\mathrm{PT}=2.1$ & $\mathrm{PT}=8.0 \%$ & $\mathrm{PT}=53.4 \%$ \\
& $\mathrm{FM}=1.7$ & $\mathrm{FM}=6.9 \%$ & $\mathrm{FM}=49.4 \%$ \\
\hline
\end{tabular}

Note. $\mathrm{PT}=$ patient $(n=110) ; \mathrm{FM}=$ family member $(n=109)$. 
Table 5: Comparison of SCI Scale Scores Patient and Family Members: Paired $t$ Test

\begin{tabular}{lcccc}
\hline & Reciprocity & Communication & Decision Making & Balance \\
\hline Patient mean score & 4.65 & 4.24 & 4.88 & 4.71 \\
Family mean score & 4.86 & 3.99 & 4.56 & 4.10 \\
$p$ value & .01 & .051 & .003 & .000 \\
\hline
\end{tabular}

Note. $n=96$ dyads. $\alpha<.05$. 


\section{Table 6: Pearson Correlations Between Shared Care and Mutuality Subscale Score}

\begin{tabular}{|c|c|c|c|c|c|}
\hline \multirow[t]{2}{*}{1.} & \multirow[t]{2}{*}{2.} & \multicolumn{4}{|c|}{ 3. Mutuality Subscale Scores } \\
\hline & & Shared Values & Love & Activity & Reciprocity \\
\hline \multirow{8}{*}{$\begin{array}{l}\text { Shared Care } \\
\text { subscales }\end{array}$} & Reciprocity & $\mathrm{PT}=.40^{* *}$ & $\mathrm{PT}=.45^{\star *}$ & $\mathrm{PT}=.49^{\star \star}$ & $\mathrm{PT}=.59^{* *}$ \\
\hline & & $\mathrm{FM}=.60^{\star *}$ & $\mathrm{FM}=.55^{\star \star}$ & $\mathrm{FM}=.55^{\star \star}$ & $\mathrm{FM}=.42^{* *}$ \\
\hline & Communication & $\mathrm{PT}=.16$ & $\mathrm{PT}=.13$ & $\mathrm{PT}=.20$ & $\mathrm{PT}=.19$ \\
\hline & & $\mathrm{FM}=.13$ & $\mathrm{FM}=.10$ & $\mathrm{FM}=.24^{*}$ & $\mathrm{FM}=.38^{* *}$ \\
\hline & Decision making & $\mathrm{PT}=.08$ & $\mathrm{PT}=.14$ & $\mathrm{PT}=.10$ & $\mathrm{PT}=.10$ \\
\hline & & $\mathrm{FM}=.31^{\star \star}$ & $\mathrm{FM}=.31^{* *}$ & $\mathrm{FM}=.39^{* *}$ & $\mathrm{FM}=.49^{* *}$ \\
\hline & Balance & $\mathrm{PT}=.05$ & $\mathrm{PT}=.13$ & $\mathrm{PT}=.07$ & $\mathrm{PT}=.18$ \\
\hline & & $\mathrm{FM}=.16$ & $\mathrm{FM}=.13$ & $\mathrm{FM}=.28^{*}$ & $\mathrm{FM}=.34^{\star *}$ \\
\hline
\end{tabular}

Note. $\mathrm{PT}=$ patient $(n=78) ; \mathrm{FM}=$ family member $(n=77)$.

${ }^{*}$ Correlation is significant at the .05 level (two-tailed). ${ }^{* *}$ Correlation is significant at the .01 level (two-tailed). 
Figure 1: Shared Care Construct

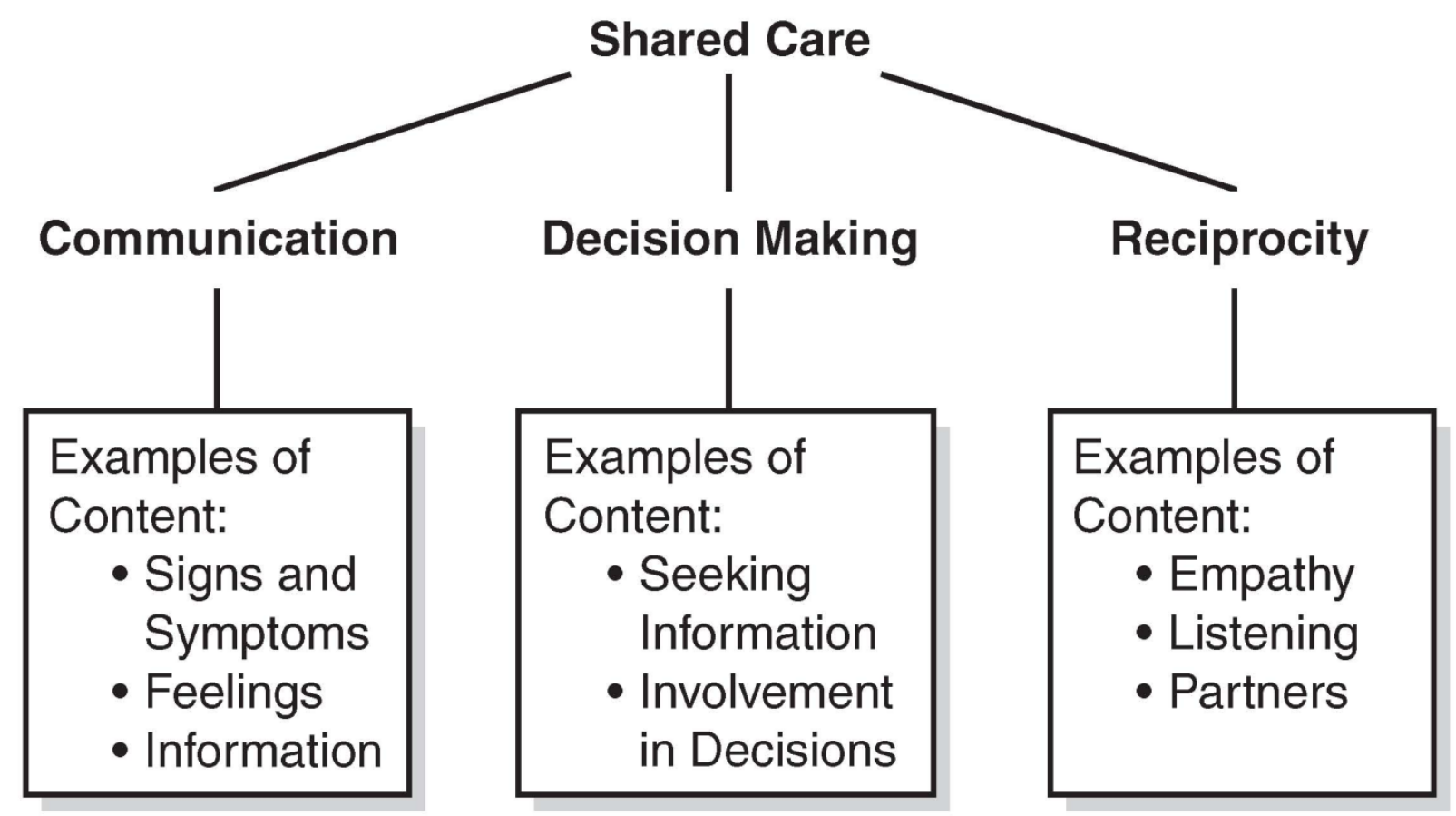

